NUCLEAR INSTRUMENTS AND METHODS II8 (1974) 43I-434; (O) NORTH-HOLLAND PUBLISHING CO.

\title{
A CALORIMETER FOR 300 GeV NEUTRONS AND PROTONS*
}

L. W. JONES, J. P. CHANOWSKI, H. R. GUSTAFSON, M. J. LONGO, P. L. SKUBIC, J. L. STONE

\author{
University of Michigan, Ann Arbor, Michigan 48104, U.S.A. \\ and \\ BRUCE CORK' \\ Argonne National Laboratory, Argonne, Illinois 60439, U.S.A.
}

Received 30 January 1974

\begin{abstract}
A total-absorption calorimeter for determining the energies of hadrons has been built and tested in beams at the National Accelerator Laboratory. The calorimeter is being used in an experiment to measure neutron total cross sections. The design of
\end{abstract}

The ionization calorimeter, or total-absorption spectrometer, has proven to be a valuable device for measuring hadron energies since its first introduction in cosmic-ray experiments by Grigorov et al. ${ }^{1}$ ). The principle of operation is based on the total absorption of the energy of an incident, high-energy hadron by a large volume of dense material, such as iron, throughout which detestors are interspersed to sample the ionization energy loss. Within this volume the incident particle interacts and generates a complex cascade of electromagnetic showers and hadrons (pions, nucleons, and nucleai fragments). This cascade process has been studied by various groups ${ }^{2-5}$ ), and experiments on calorimeter properties have been carried out using proton beams from accelerators below $30 \mathrm{GeV}^{6-8}$ ). Homogeneous detectors, in par ticular large volumes of sodium-iodide scintillator, have also been explored ${ }^{9}$ ). While many terms and names have been used for these devices, the general term calorimeter will be used in this report ${ }^{10}$ ).

The calorimeter of the present study was designed as a neutron detector in an experiment to measure total cross sections of neutrons on hydrogen and other elements between 50 and $300 \mathrm{GeV}$ at the National Accelerator Laboratory ${ }^{1}$ ). As the neutron beam has a continuous energy spectrum, the energy resolution of the calorimeter is required to measure any energy dependence of the cross sections. This calorimeter is made of alternating plates of iron and plastic scintillator, as illustrated in fig. 1. The iron is in the form of thirty plates, each $3.81 \times 61 \times 61 \mathrm{~cm}^{3}$, bolted on

\footnotetext{
- Supported by the U.S. National Science Foundation and the Atomic Energy Commission.

+ Now at the Lawrence Berksley Laboratory.
}

the calorimeter is described and its performanse is reported. The resolution of the calorimeter for $300 \mathrm{GeV}$ protons is found to be $12.8 \%$ fwhm.

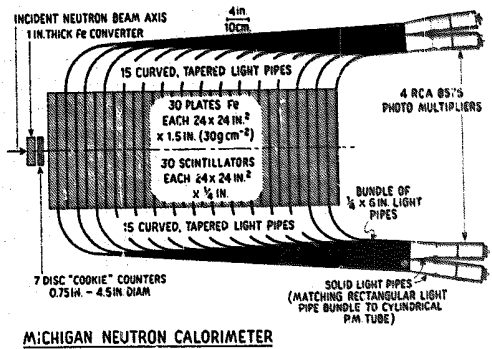

Fig. 1. Plan view of the calorimeter.

$5.08 \mathrm{~cm}$ centers to a base plate of cast aluminum. Thirty sheets of Pilot " $F$ " plastic scintillator, each $0.635 \times 61 \times 61 \mathrm{~cm}^{3}$, were sandwiched between the iron plates. Thus the calorimeter is $900 \mathrm{~g} / \mathrm{cm}^{2}$ thick in iron plus $20 \mathrm{~g} / \mathrm{cm}^{2}$ of plastic scintillator. Light is brought from the scintillators through simple light pipes of $0.635 \mathrm{~cm}$ thick lucite, tapered from 61 to $15.25 \mathrm{~cm}$, and bent through $87^{\circ}$. The $15.25 \mathrm{~cm}$ wide light pipes from the 15 scintillators on either side are brought together in a bundle to the rear of the calorimeter where matching lucite cones reduce the area to match the cathode areas of four RCA 8575 photomultipliers. These phototubes were found to be the best of various RCA and Philips photomultipliers studied, with respect to dynamic range and gain stability. Some tubes (such as the 56 DVP) displayed a gain which varied with count rate.

In order to equalize the pulse heights from the 30 
scintilators, a ${ }^{106} \mathrm{Ru}$ beta source was placed at the center of each sintillator in curn, and the corresponding light pipe was then masked so that the phototube current due to the beta source was the same to within $\pm 5 \%$ for each of the 30 scintillators.

Ahead of the calorimeter proper is located a set of seven disc-shaped scintillation counters of from 1.91 to $11.42 \mathrm{~cm}$ diameter centered on the beam axis. These are preceded $b y$ an iron converter plate normally $2.54 \mathrm{~cm}\left(20 \mathrm{~g} / \mathrm{crn}^{2}\right)$ thick. Neutrons which interacted in the iron plate produced charged particles into a forward cone which were detected in the disc counters. The pulses from these counters were then used to determine the radius from the beam axis of the interacting neutron. A $20 \mathrm{~cm}$ diameter anticoincidence counter several meters upstream of the converter insured that only incident neutral particles were detected.

The calorimeter phototubes are run with $300 \mathrm{~V}$ between the cathode and first dynode, with four "afterburner" power supplies to stabilize the voltage to the last four dynodes, and with high voltages of $1700-1800 \mathrm{~V}$ overall. The anode pulses are summed in a LeCroy model $127 \mathrm{~L}$ active fan-in and the output distributed in a model $128 \mathrm{~L}$ active fan-out to several disctiminators and to a Northern "Econ I" pulseheight analyzer. Pulses from the phototube anodes are clipped to about $18 \mathrm{~ns}$ and clipping lines are terminated with resistors to minimizi pulse overshoot. A pedestal is added to the pulse into the pulse-height analyzer in order to minimize non linearities neat threshold.

High-energy muons couid be studied by inserting a $2.4 \mathrm{~m}$ long iron block into the beam $100 \mathrm{~m}$ upstream of the calorimeter. Trigger counters upstream and downstream from the calorimeter were then used to insure that the muors passed through the calorimeter. The resulting muon pulse-height distribution in the calorimeter counters is given in fig. 2.

The neutron spectrum obtained with $300 \mathrm{GeV}$ protons incident on a beryllium target is given in fig. 3 . The peak in the spectrum corresponds to about $240 \mathrm{GeV}$. At very low energies (below $30 \mathrm{GeV}$ ) there is a large number of low-energy gamma rays. The height of this gamma peak is a strong function of the cleanliness of the proton targeting, the neutron collimator, and the placement of lead filters in the beam. Normally, the beam contains $5 \mathrm{~cm}$ of lead ahead of the first swseping magnet. From calculations and from flux measurements in charged-particle beams at larger production angles, it is probable that there is a neutral kaon component in the beam which constitutes as much as $30 \%$ of the beam near $30 \mathrm{GeV}$ but falls

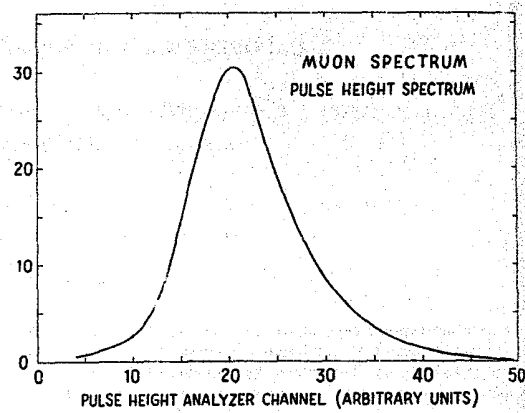

Fig. 2. A muon pulse-height spectrum taken with the calorimeter. The numbers on the abscissa scale differ by a factor of 25.3 from those of figs. 3 and 4.

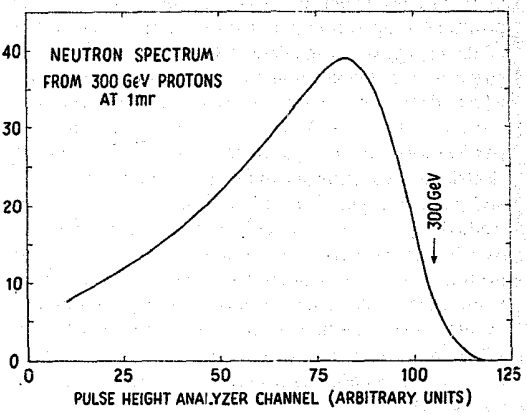

Fig. 3. A typical pulse-height spectrum of neutrons produced by $300 \mathrm{GeV}$ protons on a $12^{*}$ beryllium target at $1 \mathrm{mr}$.

rapidly to a few percent near $100 \mathrm{GeV}$ and a negligible fraction above $200 \mathrm{GeV}$.

The most interesting data relevant to the calorimeter performance are data taken with a monochromatic proton beam. This beam was made by shifting two collimators in the neutron beam and appropriately energizing bending magnets normally used as sweeping magnets in the neutron beam. The protons were primary beam protons diffraction-scattered through about $1 \mathrm{mr}$ by the Be target and the energy spread is believed to be $<1 \%$. 
In order to simulate the neutron case, protons were required to interact in the iron neutron converter ahead of the calorimeter by requiring about three times the single-particle threshold in the triggering disc counters. (Anticoincidence counters are of course removed from the logic in this case) Pulse-height spectra from 209 and $300 \mathrm{GeV}$ protons are presented in fig. 4. The $300 \mathrm{GeV}$ spectrum, taken with a $10 \mathrm{~g} / \mathrm{cm}^{2}$ iron converter plate, has a full width at half maximum of $12.8 \%$. (This broadened to $13.5 \%$ fwhm with a $20 \mathrm{~g} / \mathrm{cm}^{2}$ iron converter.) The $200 \mathrm{GeV}$ peak in fig. 4 was taken with a $20 \mathrm{~g} / \mathrm{cm}^{2}$ converter and has a $14.4 \%$ fwhm. The center of the $200 \mathrm{GeV}$ peak is quite accurately $\frac{2}{3}$ of the center of the $300 \mathrm{GeV}$ peak, confirming that the calorimeter's energy response is linear over this range of energies to within experimental uncertainties of less than $\pm 2 \%$. These experimental uncertainties arise from the relative normalization of the two pulse-height spectra.

It is of interest to make an absolute calibration of the calorimeter; that is, to be able to convert a pulse height measured relative to some lower-energy standard (such as the pulse height from muons or electrons) to an absolute hadron energy. This was done by comparing the average pulse height from muons traversing the calorimeter with the average $300 \mathrm{GeV}$ proton pulse height. The proton-muon pulse-height ratio was 120:1, with an uncertainty of $\pm 5 \%$. The energy loss of a muon traversing the calorimeter is well known from rangeenergy tables if the muon energy is known. Here the muon energy was assumed to be $10 \mathrm{GeV}$, although average energies as low as $5 \mathrm{GeV}$ and as high as

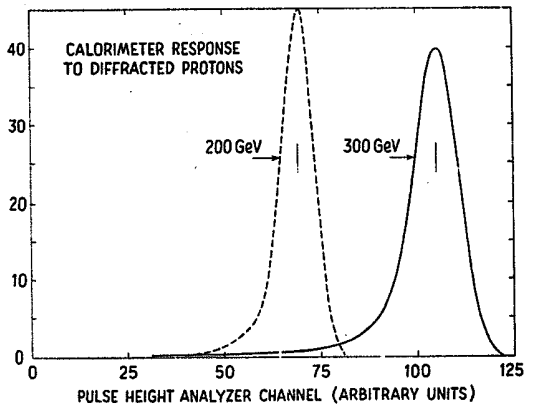

Fig. 4. The pulse-height spectra from 200 and $300 \mathrm{GeV}$ protons incident on the calorimeter.
$20 \mathrm{GeV}$ are possible. The energy loss in $3.81 \mathrm{~cm}$, or $30 \mathrm{~g} / \mathrm{cm}^{2}$, of iron by $10 \mathrm{GeV}$ muons is $58.9 \mathrm{MeV}$, and the energy loss in $0.635 \mathrm{~cm}$ of plastic scintillator is $1.81 \mathrm{MeV}$, so that the muon energy loss in each module is $60.7 \mathrm{MeV}$ or about $1.82 \mathrm{GeV}$ in the entire calorimeter ${ }^{12}$ ). This figure is uncertain by $\pm 5 \%$ if the average muon energy is between 5 and $20 \mathrm{GeV}$. From the proton-muon pulse-height ratio, protons produce pulses corresponding to $218 \mathrm{GeV}$ in "visible" energy in the calorimeter, or $(72 \pm 5) \%$ of $300 \mathrm{GeV}$. The error results from combining the uncertainty in protonmuon pulse-height ratio and the uncertainty in muon energy loss in quadrature. There are several reasons for this $28 \%$ inefficiency:

1) A fraction of the energy in collisions with iron nuclei goes into nuclear binding energy and is lost to escapirg fast neutrons.

2) Nuclear fragments and slow protons are detecied less efficiently in plastic scintillator than relativistic particles.

3) There is a "transition" effect on the detection of the electromagnetic shower component due to the difference in critical energy between iron and plastic. This results in a pulse height lower by a few percent in the scintillator for a given electromagnetic shower energy than would be the case with a homogeneous detector ${ }^{13}$ ).

4) In addition, there are undoubtedly other small effects due to the different ratios between radiation length, interaction mean free path, and $\mathrm{d} E / \mathrm{d} x$ for iron and plastic.

An absolute normalization was calculated for a calorimeter used in recent cosmic-ray experiments at Echo Lake, Colorado ${ }^{14}$ ). It is interesting in retrospect to compare with these figures. There the transition effect was calculated to contribute an average $8 \%$ loss in "visible" energy, and the nuclear effects and scintillator non-linearity $15 \%$ at $300 \mathrm{GeV}$. Consequently, the present measured figure of $(72 \pm 5) \%$ may be compared with $77 \%$ for that detector ${ }^{15}$ ).

In summary, we have been able to achieve an energy resolution of $12.8 \%$ fwhm for $300 \mathrm{GeV}$ protons with a relatively simple calorimeter. It is probable that this resolution could be further improved somewhat by using longer gate widths, elimination of the clipping of the phototube pulses, and better scintillator matching.

We would like to thank $O$. Haas and D. Koch for their excellent technical assistance and the staff of the National Accelerator Laboratory for their valuable cooperation. 


\section{References}

1) N. L. Grigorov, V. S. Murzin and I. D. Rapapo.t, J. Exptl. Theoret. Phys. 34 (1958) 506 [English transhtion in Sov. Phys. JETP 7 (1958) 348].

2) V. S. Murzin, Progress in elementary particle and cosmic roy physics (Nonth-Holland Publ. Co. Amsterdam, 1967) Vol. 9.

3) P. V. Ramana Murthy, B. V. Sreekantan, A. Subramanian and S. D. Verma, Nucl. Instr. and Meth. 23 (1963) 245.

4) E. V. Denisov et al., Intern. Conf. on Cosmic rays, (London, 1965) vol. 2, p. 824.

5) W. V. Jones, Phys. Rev, 187 (1969) 1868.

G) W. I. Jones, K. Pinkau, U. Pollvogt, W. K. H. Schmidt and R. W. Huggett. Nucl. Instr. and Meth. 72 (1969) 173.

7) H. Whiteside, C. J. Crannell, H. Crannell, J. F. Ormes, M. J. Ryan and W. V. Jones, Nucl. Instr. and Meth. 109 (1973) 375 .

๙) J. Engler, W. Flauger, B. Gibbard, F. Monnig, K. Runge and H. Schopper, Nucl. Instr. and Meth. 106 (1973) $18 \%$.

9) E. B. Hughes, R. Hofstadter, W. L. Lakin and I. Sick, Nucl. Instr. and Meth. 75 (1969) 130.

19) Our use of the term "calorimeter" has its iogical antecedent in experiments with betatrons wherein the absolute cali- bration of a bremsstrahlung X-ray beam was made by totaliy absorbing the beam in a lead cylinder and observing the temperature rise of the lead. D. W. Kerst and G. A. Price, Phys. Rev. 79 (1950) 725.

11) Expt. no. 4, National Accelerator Laboratory (May 1970).

12) W. P. Trower, Range-energy and $\mathrm{d} E / \mathrm{d} x$ plots of charged particles in matter, high-energy particle data, UCRL 2426, vol. 2 (1966 Rev.) (unpublished); Studies in penetration of charged particles in matter, Nucl. Sci. Ser. Rept. No. 39 , National Academy of Sciences - National Research Council. Pub. 1133 (1967).

13) I. N. Fetisov, Can. J. Phys. 46 (1968) S1145; C. J. Crannell, H. Crannell, C. R. Gillespie, K. Pinkau and R. R. Whitney, Phys. Rev. 182 (1969) 1435.

14) L. W. Jones et al., Nucl. Phys. B43 (1972) 477; G. D. Demeester, Thesis (Univ. of Mich, 1971) Report UM HE 71-5 (unpublished).

15) Bechuse the transition effect is relatively small in this calorimeter, the discrepancy between these results and the Echo Lake calorimeter may be greater than these figures would indicate. Based on the present calibration, it is probable that an underestimate of hadron energies by about $10 \%$ was made in the Echo Lake data. 